Article

\title{
Development of a Wearable Sensor Algorithm to Detect the Quantity and Kinematic Characteristics of Infant Arm Movement Bouts Produced across a Full Day in the Natural Environment
}

\author{
Ivan A. Trujillo-Priego ${ }^{1}$, Christianne J. Lane ${ }^{2}$, Douglas L. Vanderbilt ${ }^{3}$, Weiyang Deng ${ }^{1}$, \\ Gerald E. Loeb ${ }^{4}$, Joanne Shida ${ }^{1}$ and Beth A. Smith ${ }^{1, *}$ \\ 1 Division of Biokinesiology and Physical Therapy, University of Southern California, Los Angeles, \\ CA 90089-9006, USA; itrujill@usc.edu (I.A.T.-P.); weiyangd@usc.edu (W.D.); \\ jshida-tokeshi@olmstedcenter.org (J.S.) \\ 2 Department of Preventative Medicine, Division of Biostatistics, Keck School of Medicine, \\ University of Southern California, Los Angeles, CA 90089-9234, USA; christianne.lane@med.usc.edu \\ 3 Department of Pediatrics, Division of General Pediatrics, Keck School of Medicine, \\ University of Southern California, Los Angeles, CA 90089-9234, USA; dvanderbilt@chla.usc.edu \\ 4 Department of Biomedical Engineering, Viterbi School of Engineering, University of Southern California, \\ Los Angeles, CA 90089, USA; gloeb@usc.edu \\ * Correspondence: beth.smith@usc.edu; Tel.: +1-323-442-4072
}

Received: 21 May 2017; Accepted: 20 June 2017; Published: 23 June 2017

\begin{abstract}
We developed a wearable sensor algorithm to determine the number of arm movement bouts an infant produces across a full day in the natural environment. Full-day infant arm movement was recorded from 33 infants (22 infants with typical development and 11 infants at risk of atypical development) across multiple days and months by placing wearable sensors on each wrist. Twenty second sections of synchronized video data were used to compare the algorithm against visual observation as the gold standard for counting the number of arm movement bouts. Overall, the algorithm counted 173 bouts and the observer identified 180, resulting in a sensitivity of $90 \%$. For each bout produced across the day, we then calculated the following kinematic characteristics: duration, average and peak acceleration, average and peak angular velocity, and type of movement (one arm only, both arms for some portion of the bout, or both arms for the entire bout). As the first step toward developing norms, we present average values of full-day arm movement kinematic characteristics across the first months of infancy for infants with typical development. Identifying and quantifying infant arm movement characteristics produced across a full day has potential application in early identification of developmental delays and the provision of early intervention therapies to support optimal infant development.
\end{abstract}

Keywords: wearable sensors; infants; arm movement; movement system

\section{Introduction}

Infancy is a period of exploration and learning characterized by the development of motor skills. At least some of these skills arise from changes in synaptic connectivity that occur in response to recurring patterns of neural activity, as suggested by Hebb in the 1960s [1]. Profound behavioral changes can occur in infants as a result of both enriched [2] and deprived [3] motor experience. Extensive research demonstrates a striking relationship between the acquisition of new motor skills and subsequent cognitive development in infancy (for example [4-6]), implying that intervention to promote motor skills could be used to enhance the overall infant developmental rate. One of the biggest 
current challenges in this area, however, is accurately measuring the amount and type of movement practice infants are producing in order to identify relationships between movement practice and motor skill development. We do not currently know how much or what type of practice is necessary for an infant to learn a motor skill, for example reaching using their arms.

Wearable sensors record tri-axial accelerometer and/or gyroscope data at many samples per second, allowing us to record movement data unobtrusively across many continuous hours. We propose that wearable sensors should allow for the measurement of the amount and type of infant movement practice across days and months, unobtrusively across many continuous hours in the natural environment. To date, however, researchers have only recorded this type of data from infants for up to an hour, in a laboratory or clinical environment [7-13]. Another option is commercially available activity monitors such as Actigraph [14] and Actical [15], but they do not identify specific movement characteristics and only report relative intensity of physical activity. Although relative intensity of physical activity could be informative for certain research questions, their use has not been validated in infants. We are the first to validate the use of wearable sensors to identify and describe kinematic characteristics of infant arm movements from full-day data.

Our purpose here is to describe the development of an algorithm to identify and classify bouts of infant arm movement from wearable sensor data. To develop the algorithm, we included 33 infants (22 infants with typical development (TD) and 11 infants at risk of atypical development (AR)). We included infants with TD and AR in the development of the algorithm as our goal is to be able to use it in both populations. Some of the infants AR will eventually receive a diagnosis reflecting motor impairment, while others will not. They may or may not have detectable movement differences at this stage. We wanted to develop the algorithm to detect the movement of infants with a broad, representative range of movement characteristics to ensure that we could use one consistent algorithm process across both groups. Next, as the first step toward developing norms, we quantified full-day arm movement bouts and kinematic characteristics across the first months of infancy for infants with TD. Kinematic characteristics included duration, average and peak acceleration, average and peak angular velocity, and type of movement (one arm only, both arms for some portion of the bout, or both arms for the entire bout). Identifying and quantifying infant arm movement characteristics produced across a full day has potential applications in early identification of developmental delays and in the development and clinical testing of early intervention therapies.

\section{Materials and Methods}

\subsection{Participants}

Twenty-two infants with TD and eleven infants AR were included in this study. Infants with $\mathrm{TD}$ and AR were included in the algorithm development portion of the study in order to create an algorithm that is useful and accurate for both groups. Infants AR are not included in the presentation of average full-day values of arm movement bout characteristics. The infants AR are a small and broad group of infants with various risk levels for atypical development and a variety of developmental challenges. Their data do not lend themselves to pooling but will instead be correlated with individual future developmental outcomes. Infants with TD were from singleton, full-term (38 weeks minimum gestation) births. Infants experiencing complications during birth, or with any known visual, orthopedic, or neurologic impairment, or a score at or below the 5th percentile for their age on the Bayley Scales of Infant Development (3rd edition) [16] at the time of testing were excluded from the TD group. Infants in this group were between 38 and 203 days of age. Infants AR were born before 36 weeks of gestation or defined as at high risk for developmental delay per the definition of the state of California [17]. Infants AR were a broad group and included infants who were born small for gestational age, had congenital heart defects, or had known neurologic malformations, for example. Infants in this group were between 40 and 230 days (adjusted for prematurity). This study was approved by the Institutional Review Board of the University of Southern California. Infants 
were recruited at health care clinics and by word of mouth in the Los Angeles area. A parent or legal guardian signed an informed consent form for their infants' participation.

\subsection{Procedures}

Infants were measured once per month, from 1 to 6 visits each. We traveled to the infants' home in the morning. Continuous full day arm movement was recorded using two battery-powered wearable sensors (Opals, APDM, Inc., Portland, OR, USA), one on each wrist. Each sensor contains a tri-axial accelerometer, gyroscope, and magnetometer, and was placed inside a pocket in a custom wrist band (see Figure 1). The sensors were actively synchronized to each other, recording at 20 samples per second. Recordings began in the morning and continued until the infant was put to bed for the night. At this point, the caregiver removed the sensors, resulting in anywhere from 8 to $13 \mathrm{~h}$ of continuous data. Caregivers were encouraged to perform their typical daily activities.

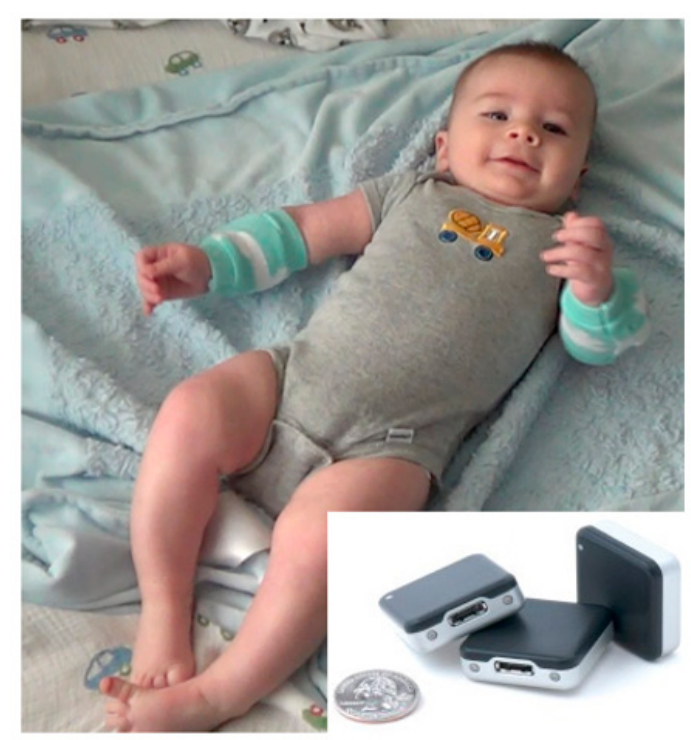

Figure 1. Infant with typical development (104 days of age) with sensors on right and left arms. Sensors are shown in inset with a U.S. quarter for reference.

Once the wearable sensors were on for each assessment, we recorded five minutes of video of the infants' spontaneous movements in the supine position. We assessed development using the Bayley Scales of Infant Development (3rd edition) [16] and the Alberta Infant Motor Scale [18]. We also measured weight, length, head circumference, and upper and lower extremity lengths and circumferences. We also collected approximately $10 \mathrm{~min}$ of electroencephalography data during an assessment of arm reaching skill, however those results are not presented here.

\subsection{Algorithm Development}

We developed a threshold-based algorithm in Matlab to identify infant arm movement bouts from the wearable sensor data for acceleration and angular velocity. The first step was to define general rejection thresholds for the acceleration and angular velocity values, which were the same for all infants. Then thresholds indicative of movement bouts were determined separately and uniquely for each infant according to a standard formula that identified local maxima within the complete sensor record. These thresholds should be computed from the data from each infant to compensate for differences in infant size and sensor placement. They assure that bouts reflect real, purposeful movements by the infant rather than background noise and small movements such as positional shifts. Movement bouts were identified as periods when both the acceleration and velocity exceeded these unique thresholds. 


\subsection{Acceleration Rejection Threshold Determination. Detrending and Rectification}

First, we calculated resultant linear acceleration as the square root squared sum of each axis $(x, y, z)$ as follows:

$$
\text { Accel }_{\text {mag }}=\sqrt{\mathrm{Ax}_{\mathrm{x}}^{2}+\mathrm{A}_{\mathrm{y}}^{2}+\mathrm{A}_{\mathrm{z}}^{2}}
$$

Next, the resultant acceleration was detrended by subtracting the median to remove the gravity component and any steady noise or offsets embedded in the signal. Then we used synchronized video and sensor data (for randomly selected infants) to empirically determine a general rejection threshold for acceleration values. The detrended acceleration signal was then rectified by applying the general rejection threshold of $\mathrm{a}=[-1.02,1.32] \mathrm{m} / \mathrm{s}^{2}$ (Figure 2 top).
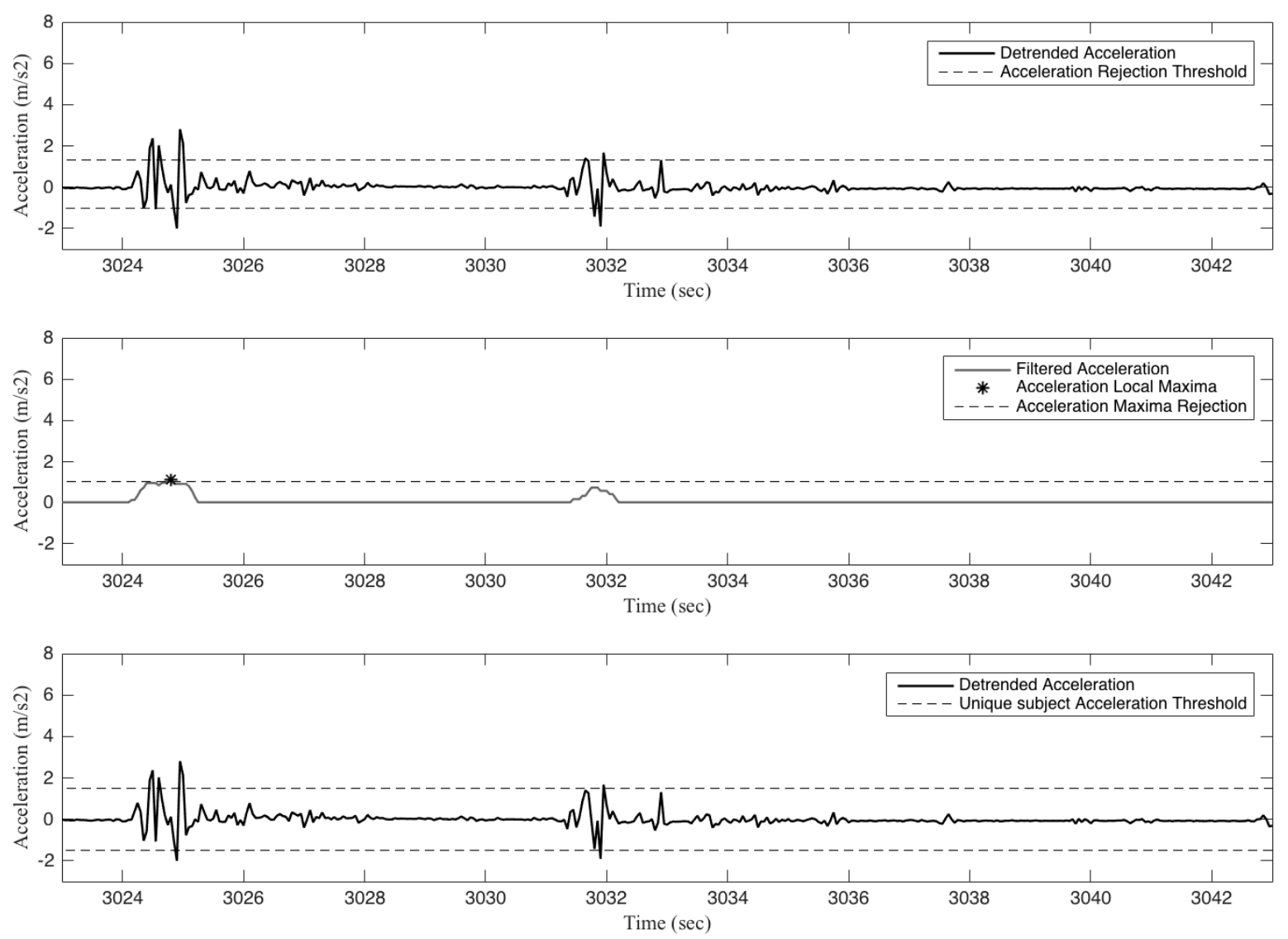

Figure 2. The top panel shows a representative detrended Accel mag signal (solid black line) and the general rejection thresholds (black dashed lines $a=[-1.02,1.32] \mathrm{m} / \mathrm{s}^{2}$ ) for a $20 \mathrm{~s}$ section from the right arm of a 3-month-old infant with typical development. The middle panel shows the residual acceleration signal after full-wave rectification and smoothing (moving average with a $0.5 \mathrm{~s}$ window, solid black line) and the identification of local maxima (asterisk) for the filtered signal by disregarding maxima below values of $1.0 \mathrm{~m} / \mathrm{s}^{2}$ (dashed black line). The bottom panel shows the same representative detrended acceleration signal (solid black line) with this participant's unique acceleration threshold $\pm 1.4972 \mathrm{~m} / \mathrm{s}^{2}$ (dashed black lines).

\subsection{Filtering and Threshold Determination}

The signal remaining after rejection was full-wave rectified and smoothed with a moving average filter $(0.5 \mathrm{~s}$ window). We computed a unique bout threshold for each participant to distinguish potentially bout-related acceleration values from smaller movements, and we identified local maxima for the filtered signal by disregarding maxima below values of $1.0 \mathrm{~m} / \mathrm{s}^{2}$ (Figure 2 middle). We then set the infant's unique acceleration threshold as the mean of all such local maxima minus half standard 
deviation for both positive and negative values (Figure 2 bottom). Both positive and negative values of detrended acceleration that exceeded this bout threshold were used to decide when a bout had occurred. The range of unique acceleration thresholds across infants was from -2.3604 to $-1.3113 \mathrm{~m} / \mathrm{s}^{2}$ and 1.3113 to $2.3604 \mathrm{~m} / \mathrm{s}^{2}$, for negative and positive thresholds, respectively.

\subsection{Angular Velocity Rejection Threshold Determination. Detrending and Rectification}

Similar to the acceleration signal, first, we calculated the angular velocity $(\omega)$ magnitude:

$$
\text { Ang vel }{ }_{\text {mag }}=\sqrt{\omega_{\mathrm{x}}^{2}+\omega_{\mathrm{y}}^{2}+\omega_{\mathrm{z}}^{2}}
$$

Next, the resultant angular velocity was detrended with the median to remove steady background noise embedded in the signal. We used synchronized video and sensor data (for randomly selected infants) to empirically determine a general rejection threshold for angular velocity as $\mathrm{w}=0.32 \mathrm{rad} / \mathrm{s}$ (Figure 3 top).
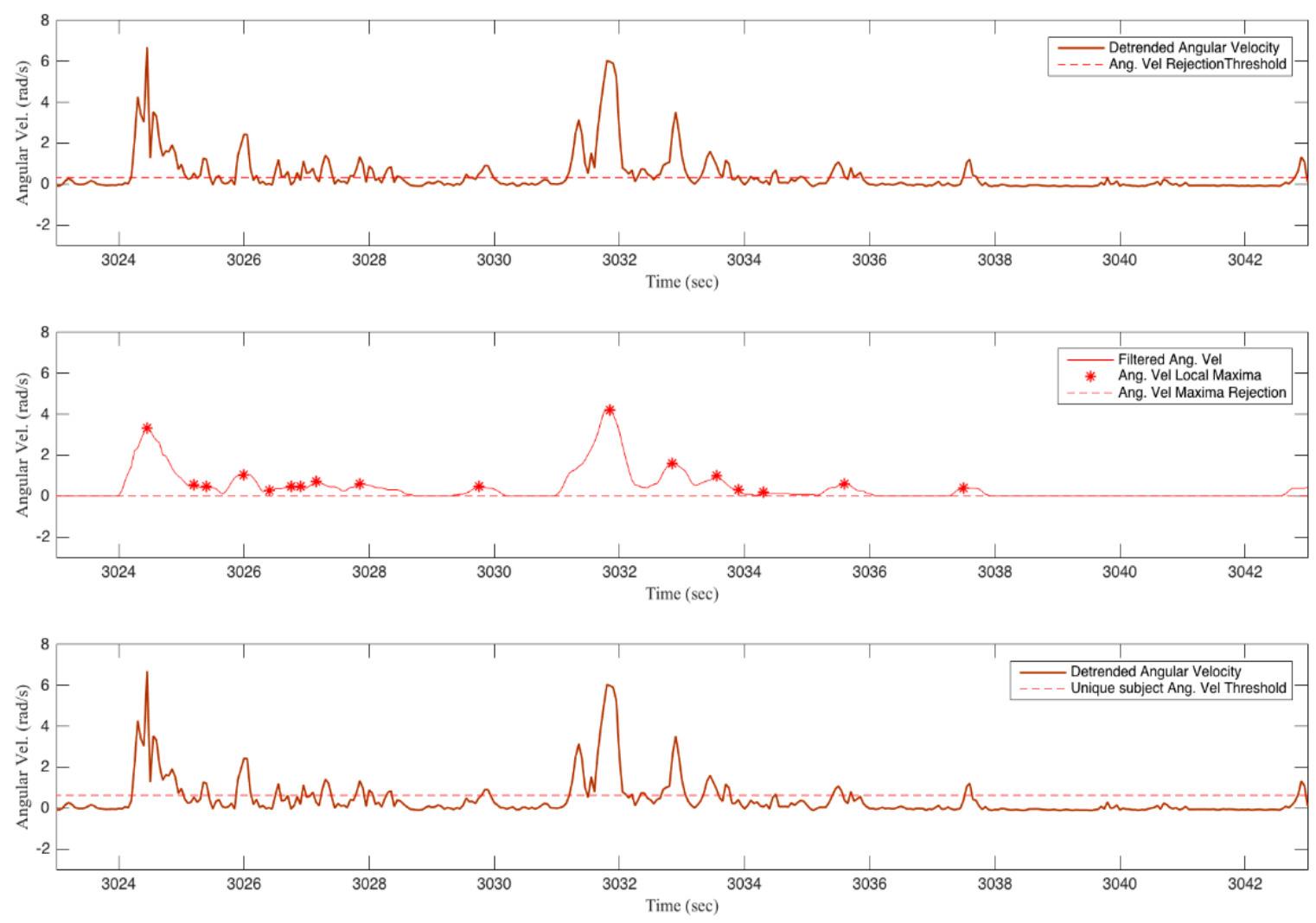

Figure 3. The top panel shows a representative Ang vel mag signal (red solid line) and the general angular velocity rejection threshold (red dashed line at $\mathrm{w}=0.32 \mathrm{rad} / \mathrm{s}$ ) for a $20 \mathrm{~s}$ section of data from the right arm of a 3-month-old infant with typical development. The middle panel shows a representative filtered angular velocity signal (moving average filter with a $0.5 \mathrm{~s}$ window, solid red line) and angular velocity maxima rejection threshold of $0.01 \mathrm{rad} / \mathrm{s}$ (dashed red light line). The red asterisks represent the identified local maxima. The bottom panel shows the same representative detrended angular velocity signal (solid red line) and the participant's unique angular velocity threshold of $0.6200 \mathrm{rad} / \mathrm{s}$ (dashed red line).

\subsection{Filtering and Bout Threshold Determination}

The detrended angular velocity signal was rectified with the rejection threshold $w$, and then filtered with a $0.5 \mathrm{~s}$ window moving average filter. To determine the unique participant-based angular 
velocity threshold, we identified local maxima for the filtered signal by disregarding maxima below values of $0.01 \mathrm{rad} / \mathrm{s}$ (Figure 3 middle). We then set the unique angular velocity threshold for each participant as the mean of these local maxima minus half standard deviation (Figure 3 bottom). The range of unique angular velocity thresholds was from 0.4208 to $1.0515 \mathrm{rad} / \mathrm{s}$.

\subsection{Arm Movement Bout Detection}

A bout was defined as the period between the start and stop of significant arm movement regardless of the position, orientation, or direction of the arm and movement. The start of a bout of

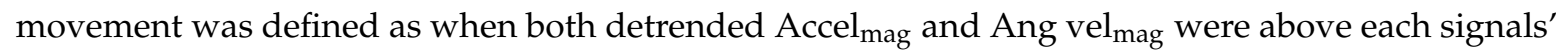
unique bout threshold. The end of a bout of movement was defined once the angular velocity magnitude went below its threshold (See Figure 4). To compare between visits, we normalized the number of bouts to the number of hours that the infant was awake and wearing the sensors. We visually estimated the amount of sleep time by identifying sleep time as periods of less than 3 movement bouts in $5 \mathrm{~min}$. This adjusted for the different lengths of sensor wear and different amounts of nap time at each visit.
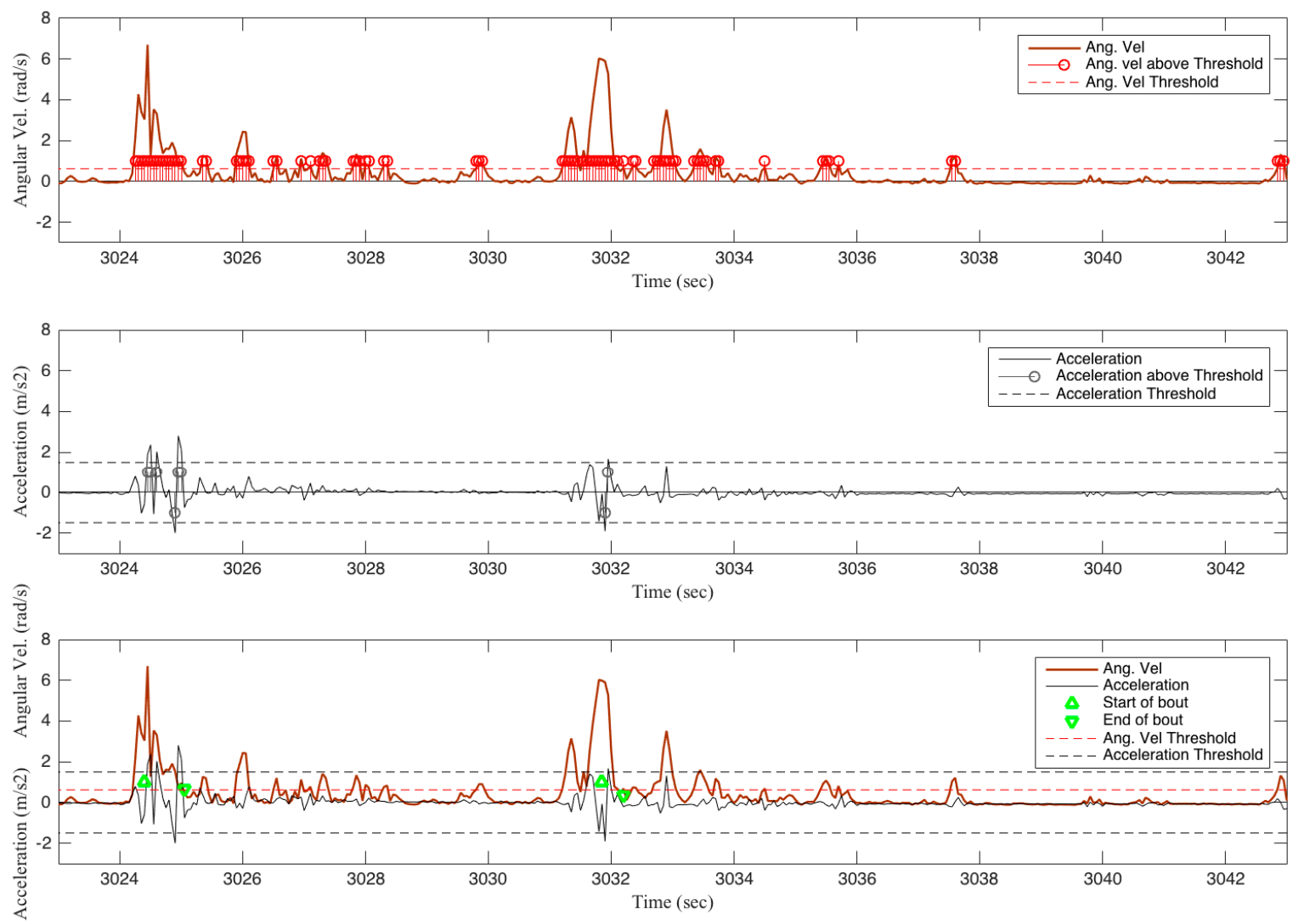

Figure 4. Top panel shows $20 \mathrm{~s}$ of the angular velocity detrended signal (rad/s solid dark red line), and the identification of values above the angular velocity threshold (light red circles; threshold dashed red line). Middle panel shows $20 \mathrm{~s}$ of the detrended acceleration signal ( $\mathrm{m} / \mathrm{s}^{2}$ solid black line), and the identification of values above the acceleration threshold (light black circles; acceleration thresholds dashed black lines). Bottom panel shows the arm movement bout count from $20 \mathrm{~s}$ of representative acceleration ( $\mathrm{m} / \mathrm{s}^{2}$; solid black line) and angular velocity ( $\mathrm{rad} / \mathrm{s}$; solid red line) data. There are two arm movement bouts identified by the algorithm. The start of each bout is identified by a green triangle pointing up in the figure when the acceleration and angular velocity each went above their unique thresholds (dashed lines). Green triangles pointing down represent the end of an arm movement bout, when the angular velocity magnitude went below its threshold. All figures are from data of the right arm of a 3-month-old infant with typical development. 


\subsection{Algorithm Performance: Counting Number of Bouts of Arm Movement}

The algorithm was compared to visual observation as the gold standard. For 10 visits from 10 different infants in each group, an interval of $20 \mathrm{~s}$ of infant arm movement was selected. We compared the number of bouts counted by the algorithm to the number identified by one expert observer (Beth A. Smith). Frame-by-frame video coding software (ELAN, The Language Archive, Nijmegen, The Netherlands) was used for the counting of movements, however for a new bout of movement to be counted each time the arm paused, the pause had to be observed in real time.

\subsection{Type of Movement}

For each arm separately, we compared the start and stop samples of each bout to those of the other arm to determine if only one arm was moving, both arms were moving for some portion of the bout, or both arms were moving for the entire bout.

\subsection{Kinematic Characteristics}

Duration (s), was determined by counting the number of samples for each bout and dividing that number by 20 , as data were collected at 20 samples per second. Average Acceleration $\left(\mathrm{m} / \mathrm{s}^{2}\right)$ was calculated as the sum of the absolute values of acceleration divided by the number of samples of the bout. Peak Acceleration $\left(\mathrm{m} / \mathrm{s}^{2}\right)$ was calculated as the maximum absolute value of acceleration within a bout.

\subsection{Acceleration Area}

As a general calculation of overall arm "activity", we also calculated the area under the absolute value of the resultant acceleration curve (Acceleration Area) across the time period that the sensors were worn by the infant. To compare between visits, we normalized the area to the number of hours that the infant was awake and wearing the sensors, rounded to the nearest five minutes. A larger normalized acceleration area value indicates that the infant was moving the arm more frequently and/or faster than a smaller value.

\subsection{Statistical Analyses}

To examine the characteristics of full-day arm movement bouts across the first months of infancy for infants with TD, trends were developed using longitudinal linear effects modeling centered at mean age. Random differences in level were estimated. There was insufficient data to allow for the estimation of individual differences in slopes, so average slopes were estimated. Models were compared using measures of fit (BIC, -2Ln Likelihood difference) in order to determine if a linear or quadratic model fit better for any given variable.

\section{Results}

\subsection{Algorithm Performance}

The algorithm was compared to visual observation as the gold standard from 20 video segments of $20 \mathrm{~s}$ each, as described previously in Section 2.9. Overall, the algorithm counted 173 bouts and the observer identified 180, resulting in a sensitivity of $90 \%$ (sensitivity $=$ true positive / (true positive + false negative)). For the infants with TD, the algorithm counted 93 bouts of arm movement and the observer identified 90 . The algorithm undercounted (did not identify a movement the observer did) by 10 and overcounted (identified a movement the observer did not) by 13 bouts. For infants AR, the algorithm counted 80 bouts of arm movement and the observer identified 90 . The algorithm undercounted by 11 and overcounted by 1 bout. Algorithm overcounting most often occurs because the algorithm is able to identify pauses in arm motion that can be as short as $1 / 20$ of a second, so the algorithm identifies 2 bouts when an observer only sees 1 . Algorithm undercounting most often happens when an infant 
produces a very long, slow arm movement (e.g., lowering one arm very slowly while focusing attention on the other arm). We did not actively identify periods of no movement, we assumed the infant was not moving if no active movement was identified by the algorithm, and therefore only report the ability of our algorithm to count movements and not its ability to count non-movement.

\subsection{Number of Bouts}

Table 1 shows the total number of bouts for the right and left arms across a full day at each visit for infants with TD. A full day ranged from 8 to $13 \mathrm{~h}$, and included variable amounts of nap time, therefore each infants' hours of awake time at each visit is also provided. To allow for the comparison of movement rates between infants and across visits, Figure 5 shows the average number of bouts per hour of awake time for the right and left arms, by age, for infants with TD. For the number of bouts per hour of awake time, infants generally move their arms more as they get older. For each arm a linear trend fit best.
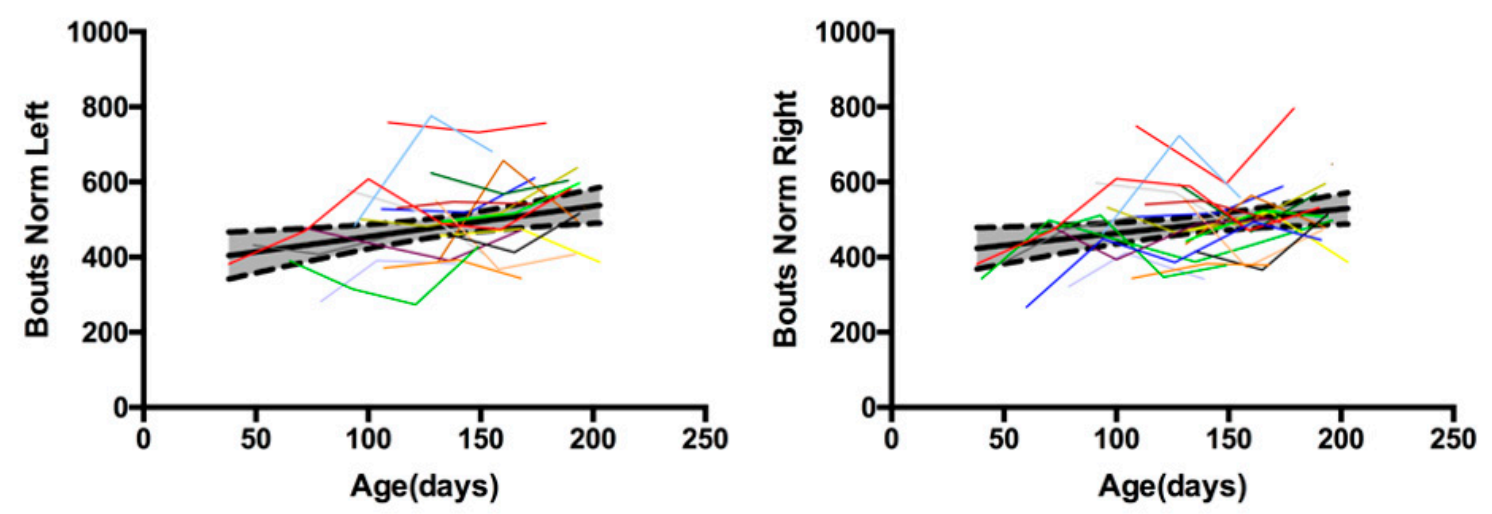

Figure 5. Average number of bouts per hour of awake time for the left and right arms, by age, for infants with typical development. Each colored line represents a different infant across 3 to 6 visits. Two single assessments are represented by dots. Thick black line is the mean and shaded area bordered by dashed black line is one standard deviation. For each arm, a linear trend across time best fit the data. 
Table 1. Overall arm movement activity, number of bouts, and type of bouts, by infant and visit.

\begin{tabular}{|c|c|c|c|c|c|c|c|c|c|c|c|c|c|}
\hline Infant & Visit & $\begin{array}{c}\text { Age } \\
\text { (Days) }\end{array}$ & $\begin{array}{c}\text { Awake } \\
\text { Time } \\
\text { (Hours) }\end{array}$ & $\begin{array}{l}\text { Acceleration } \\
\text { Area Left }\end{array}$ & $\begin{array}{l}\text { Acceleration } \\
\text { Area Right }\end{array}$ & $\begin{array}{c}\text { Total } \\
\text { Bouts Left } \\
\text { (Number) }\end{array}$ & $\begin{array}{l}\text { Bout Type: } \\
\text { Left Only } \\
\text { (Number) }\end{array}$ & $\begin{array}{l}\text { Bout Type: Left } \\
\text { with Right for } \\
\text { Portion of Bout } \\
\text { (Number) }\end{array}$ & $\begin{array}{l}\text { Bout Type: Left } \\
\text { with Right for } \\
\text { Entire Bout } \\
\text { (Number) }\end{array}$ & $\begin{array}{c}\text { Total } \\
\text { Bouts } \\
\text { Right } \\
\text { (Number) }\end{array}$ & $\begin{array}{l}\text { Bout Type: } \\
\text { Right Only } \\
\text { (Number) }\end{array}$ & $\begin{array}{c}\text { Bout Type: } \\
\text { Right with Left } \\
\text { for Portion of } \\
\text { Bout (Number) }\end{array}$ & $\begin{array}{c}\text { Bout Type: } \\
\text { Right with Lef } \\
\text { for Entire Bout } \\
\text { (Number) }\end{array}$ \\
\hline \multirow{5}{*}{1} & 1 & 60 & 9.3 & 55,715 & 55,419 & 2326 & 1126 & 790 & 410 & 2491 & 1288 & 781 & 422 \\
\hline & 2 & 94 & 6.1 & 109,295 & 101,822 & 2783 & 1195 & 987 & 601 & 2758 & 1216 & 1034 & 508 \\
\hline & 3 & 126 & 9.3 & 102,163 & 110,379 & 3242 & 1443 & 1169 & 630 & 3591 & 1774 & 1201 & 616 \\
\hline & 4 & 161 & 8.4 & 243,714 & 154,263 & 4019 & 1584 & 1695 & 740 & 4140 & 1568 & 1595 & 977 \\
\hline & 5 & 191 & 9.0 & 204,974 & 176,775 & 4001 & 1662 & 1512 & 827 & 4002 & 1656 & 1555 & 791 \\
\hline \multirow{3}{*}{2} & 1 & 113 & 8.0 & 194,156 & 172,301 & 4224 & 1648 & 1750 & 826 & 4308 & 1654 & 1597 & 1057 \\
\hline & 2 & 138 & 7.2 & 164,813 & 185,299 & 3919 & 1358 & 1596 & 965 & 3939 & 1482 & 1617 & 840 \\
\hline & 3 & 173 & 7.2 & 194,881 & 151,906 & 3904 & 1674 & 1314 & 916 & 3558 & 1389 & 1438 & 731 \\
\hline \multirow{3}{*}{3} & 1 & 128 & 8.6 & 296,547 & 310,820 & 5382 & 1887 & 2113 & 1382 & 5112 & 1753 & 2290 & 1069 \\
\hline & 2 & 160 & 8.2 & 296,786 & 280,371 & 4636 & 1870 & 1860 & 906 & 3820 & 1133 & 1778 & 909 \\
\hline & 3 & 189 & 8.1 & 332,616 & 297,343 & 4885 & 2147 & 1832 & 906 & 4604 & 1866 & 1754 & 984 \\
\hline \multirow{3}{*}{4} & 1 & 130 & 8.0 & 216,062 & 255,311 & 4372 & 1712 & 1698 & 962 & 4410 & 1799 & 1726 & 885 \\
\hline & 2 & 158 & 5.9 & 122,224 & 102,956 & 2166 & 874 & 877 & 415 & 2196 & 811 & 858 & 527 \\
\hline & 3 & 192 & 5.0 & 139,128 & 97,527 & 2030 & 775 & 857 & 398 & 2355 & 1066 & 803 & 486 \\
\hline \multirow{3}{*}{5} & 1 & 131 & 8.0 & 152,452 & 164,041 & 3944 & 1555 & 1379 & 1010 & 3537 & 1396 & 1490 & 651 \\
\hline & 2 & 165 & 5.9 & 184,351 & 132,371 & 3051 & 1114 & 1279 & 658 & 3099 & 1165 & 1242 & 692 \\
\hline & 3 & 194 & 8.4 & 233,834 & 167,870 & 5020 & 2201 & 1666 & 1153 & 4266 & 1664 & 1802 & 800 \\
\hline \multirow{3}{*}{6} & 1 & 94 & 8.1 & 214,226 & 162,435 & 3917 & 1444 & 1632 & 841 & 3746 & 1314 & 1596 & 836 \\
\hline & 2 & 128 & 8.1 & 373,701 & 240,609 & 6320 & 2393 & 2674 & 1253 & 5902 & 1965 & 2483 & 1454 \\
\hline & 3 & 155 & 8.7 & 329,202 & 344,689 & 5921 & 2740 & 1909 & 1272 & 4860 & 1874 & 2029 & 957 \\
\hline \multirow{3}{*}{7} & 1 & 79 & 6.4 & 259,136 & 224,860 & 1790 & 588 & 697 & 505 & 2050 & 868 & 805 & 377 \\
\hline & 2 & 104 & 5.5 & 145,214 & 95,116 & 2148 & 828 & 849 & 471 & 2260 & 986 & 886 & 388 \\
\hline & 3 & 139 & 7.0 & 132,645 & 117,248 & 2672 & 1099 & 950 & 623 & 2382 & 915 & 1052 & 415 \\
\hline \multirow{3}{*}{8} & 1 & 49 & 7.3 & 212,830 & 146,430 & 3138 & 1628 & 928 & 582 & 2794 & 1299 & 930 & 565 \\
\hline & 2 & 78 & 7.9 & 175,388 & 166,622 & 3229 & 1527 & 1169 & 533 & 3762 & 1943 & 1186 & 633 \\
\hline & 3 & 108 & 8.6 & 316,156 & 315,490 & 3913 & 1694 & 1417 & 802 & 4377 & 2137 & 1477 & 763 \\
\hline \multirow{3}{*}{9} & 1 & 132 & 5.7 & 207,620 & 181,761 & 2618 & 1004 & 1128 & 486 & 2704 & 965 & 1021 & 718 \\
\hline & 2 & 168 & 9.3 & 301,681 & 381,848 & 4439 & 1697 & 1658 & 1084 & 4867 & 1966 & 1694 & 1207 \\
\hline & 3 & 203 & 7.0 & 157,550 & 125,030 & 2723 & 1300 & 820 & 603 & 2727 & 1345 & 928 & 454 \\
\hline \multirow{6}{*}{10} & 1 & 40 & 8.1 & 144,204 & 154,457 & 2963 & 1302 & 1001 & 660 & 2769 & 1141 & 1003 & 625 \\
\hline & 2 & 70 & 8.8 & 281,301 & 326,961 & 4204 & 1659 & 1604 & 941 & 4397 & 1895 & 1638 & 864 \\
\hline & 3 & 97 & 8.1 & 257,067 & 274,005 & 3676 & 1397 & 1420 & 859 & 3677 & 1403 & 1432 & 842 \\
\hline & 4 & 135 & 7.5 & 137,842 & 166,236 & 2734 & 956 & 1094 & 684 & 2899 & 1138 & 1128 & 633 \\
\hline & 5 & 162 & 7.5 & 194,881 & 225,728 & 2842 & 1187 & 957 & 698 & 3262 & 1652 & 1118 & 492 \\
\hline & 6 & 196 & 8.5 & 368,220 & 311,846 & 4006 & 1635 & 1503 & 868 & 4220 & 1813 & 1604 & 803 \\
\hline \multirow{3}{*}{11} & 1 & 131 & 8.4 & 223,325 & 153,311 & 3267 & 1188 & 1426 & 653 & 3658 & 1487 & 1396 & 775 \\
\hline & 2 & 160 & 8.9 & 350,880 & 319,955 & 5857 & 2871 & 1948 & 1038 & 5025 & 2063 & 1936 & 1026 \\
\hline & 3 & 193 & 8.8 & 202,446 & 185,489 & 4376 & 2102 & 1429 & 845 & 4207 & 2004 & 1548 & 655 \\
\hline
\end{tabular}


Table 1. Cont.

\begin{tabular}{|c|c|c|c|c|c|c|c|c|c|c|c|c|c|}
\hline Infant & Visit & $\begin{array}{c}\text { Age } \\
\text { (Days) }\end{array}$ & $\begin{array}{c}\text { Awake } \\
\text { Time } \\
\text { (Hours) }\end{array}$ & $\begin{array}{c}\text { Acceleration } \\
\text { Area Left }\end{array}$ & $\begin{array}{c}\text { Acceleration } \\
\text { Area Right }\end{array}$ & $\begin{array}{c}\text { Total } \\
\text { Bouts Left } \\
\text { (Number) }\end{array}$ & $\begin{array}{l}\text { Bout Type: } \\
\text { Left Only } \\
\text { (Number) }\end{array}$ & $\begin{array}{l}\text { Bout Type: Left } \\
\text { with Right for } \\
\text { Portion of Bout } \\
\text { (Number) }\end{array}$ & $\begin{array}{l}\text { Bout Type: Left } \\
\text { with Right for } \\
\text { Entire Bout } \\
\text { (Number) }\end{array}$ & $\begin{array}{c}\text { Total } \\
\text { Bouts } \\
\text { Right } \\
\text { (Number) }\end{array}$ & $\begin{array}{c}\text { Bout Type: } \\
\text { Right Only } \\
\text { (Number) }\end{array}$ & $\begin{array}{c}\text { Bout Type: } \\
\text { Right with Left } \\
\text { for Portion of } \\
\text { Bout (Number) }\end{array}$ & $\begin{array}{l}\text { Bout Type: } \\
\text { Right with Left } \\
\text { for Entire Bout } \\
\text { (Number) }\end{array}$ \\
\hline \multirow{3}{*}{12} & 1 & 91 & 6.5 & 164,559 & 133,873 & 3767 & 1159 & 1518 & 1090 & 3894 & 1193 & 1627 & 1074 \\
\hline & 2 & 126 & 6.7 & 190,355 & 147,707 & 3435 & 1117 & 1490 & 828 & 3807 & 1441 & 1553 & 813 \\
\hline & 3 & 153 & 8.3 & 266,442 & 274,558 & 3325 & 1202 & 1433 & 690 & 3962 & 1816 & 1485 & 661 \\
\hline \multirow{4}{*}{13} & 1 & 96 & 8.3 & 205,658 & 181,497 & 4170 & 1420 & 1772 & 978 & 4424 & 1698 & 1820 & 906 \\
\hline & 2 & 126 & 4.1 & 121,324 & 71,953 & 1964 & 672 & 819 & 473 & 1901 & 629 & 824 & 448 \\
\hline & 3 & 159 & 3.3 & 259,972 & 122,373 & 1693 & 498 & 607 & 588 & 1613 & 596 & 711 & 306 \\
\hline & 4 & 193 & 3.6 & 141,730 & 129,298 & 2278 & 746 & 889 & 643 & 2125 & 652 & 978 & 495 \\
\hline \multirow{3}{*}{14} & 1 & 106 & 7.6 & 171,517 & 123,718 & 3983 & 1464 & 1533 & 986 & 3821 & 1474 & 1697 & 650 \\
\hline & 2 & 145 & 10.0 & 307,196 & 257,059 & 5210 & 1903 & 2111 & 1196 & 5193 & 1917 & 2155 & 1121 \\
\hline & 3 & 174 & 8.6 & 294,043 & 234,330 & 5255 & 2066 & 2109 & 1080 & 5063 & 1914 & 2180 & 969 \\
\hline \multirow{3}{*}{15} & 1 & 109 & 7.6 & 293,670 & 281,731 & 5743 & 2102 & 2281 & 1360 & 5671 & 2125 & 2271 & 1275 \\
\hline & 2 & 149 & 9.2 & 336,525 & 208,733 & 6774 & 3140 & 2409 & 1225 & 5499 & 1945 & 2263 & 1291 \\
\hline & 3 & 179 & 8.9 & 330,367 & 333,966 & 6769 & 2645 & 2827 & 1297 & 7113 & 2893 & 2733 & 1487 \\
\hline \multirow{4}{*}{16} & 1 & 65 & 10.1 & 154,081 & 120,320 & 3958 & 2104 & 1295 & 559 & 4680 & 2781 & 1231 & 668 \\
\hline & 2 & 93 & 7.5 & 82,914 & 129,435 & 2353 & 951 & 971 & 431 & 3826 & 2240 & 938 & 648 \\
\hline & 3 & 121 & 8.4 & 170,686 & 129,842 & 2309 & 846 & 990 & 473 & 2923 & 1363 & 1041 & 519 \\
\hline & 4 & 149 & 9.2 & 222,313 & 338,706 & 3953 & 2124 & 1225 & 604 & 3497 & 1584 & 1114 & 799 \\
\hline \multirow{6}{*}{17} & 1 & 38 & 10.4 & 186,933 & 204,148 & 3987 & 1595 & 1576 & 816 & 3989 & 1520 & 1482 & 987 \\
\hline & 2 & 72 & 10.4 & 327,534 & 269,737 & 4910 & 1832 & 1960 & 1118 & 4937 & 1785 & 1982 & 1170 \\
\hline & 3 & 100 & 9.9 & 338,695 & 320,021 & 6006 & 2242 & 2594 & 1170 & 6017 & 2145 & 2406 & 1466 \\
\hline & 4 & 133 & 8.3 & 238,994 & 219,733 & 4068 & 1385 & 1832 & 851 & 4894 & 2031 & 1829 & 1034 \\
\hline & 5 & 159 & 9.8 & 319,265 & 362,508 & 4659 & 1735 & 1788 & 1136 & 4634 & 1803 & 1768 & 1063 \\
\hline & 6 & 190 & 9.4 & 328,468 & 314,207 & 5478 & 2402 & 2028 & 1048 & 4995 & 1939 & 1995 & 1061 \\
\hline \multirow{3}{*}{18} & 1 & 107 & 10.3 & 158,525 & 173,526 & 3828 & 1916 & 1139 & 773 & 3548 & 1716 & 1336 & 496 \\
\hline & 2 & 140 & 7.6 & 149,758 & 119,284 & 3002 & 1482 & 1015 & 505 & 2922 & 1351 & 1048 & 523 \\
\hline & 3 & 168 & 9.6 & 231,146 & 188,059 & 3287 & 1543 & 1213 & 531 & 3622 & 1833 & 1133 & 656 \\
\hline \multirow{3}{*}{19} & 1 & 135 & 11.5 & 259,696 & 202,839 & 5313 & 2675 & 1607 & 1031 & 4768 & 2175 & 1687 & 906 \\
\hline & 2 & 165 & 9.7 & 220,708 & 153,669 & 4602 & 1931 & 1379 & 677 & 4580 & 1457 & 1307 & 767 \\
\hline & 3 & 194 & 8.9 & 207,079 & 199,449 & 3987 & 2188 & 1544 & 870 & 3531 & 2201 & 1616 & 763 \\
\hline \multirow{4}{*}{20} & 1 & 72 & 9.0 & 174,939 & 163,847 & 4287 & 1908 & 1563 & 816 & 4296 & 1918 & 1546 & 832 \\
\hline & 2 & 100 & 9.0 & 158,163 & 147,436 & 3960 & 2012 & 1264 & 684 & 3567 & 1655 & 1292 & 620 \\
\hline & 3 & 136 & 8.7 & 142,320 & 146,028 & 3386 & 1215 & 1471 & 700 & 4241 & 1980 & 1515 & 746 \\
\hline & 4 & 168 & 7.9 & 174,443 & 164,425 & 3721 & 1783 & 1368 & 570 & 3956 & 1964 & 1341 & 651 \\
\hline 21 & 1 & 196 & 8.4 & 289,408 & 294,277 & 4467 & 1852 & 1830 & 785 & 5430 & 2607 & 1749 & 1074 \\
\hline 22 & 1 & 203 & 10.0 & 211,167 & 188,905 & 4903 & 2054 & 1896 & 953 & 5288 & 2274 & 1876 & 1138 \\
\hline
\end{tabular}




\subsection{Type of Movement}

Table 1 shows the total number of each type of bout produced across the full day. To compare between infants and across visits, Figure 6 shows the average percentage of each type of bout produced across the full day for the right and left arms, by age, for infants with TD. In general, it appears that infants show a " $U$ "-shaped developmental trajectory for one arm only bouts, an inverted " $U$ "-shaped developmental trajectory for both arms moving for some portion of the bout, and no change across time for both arms moving for the entire bout. Quadratic trends were the best fit for one arm only and both arms moving for some portion of the bout for the right and left arms, while a linear trend best fit both arms moving for the entire bout for the right and left arms.
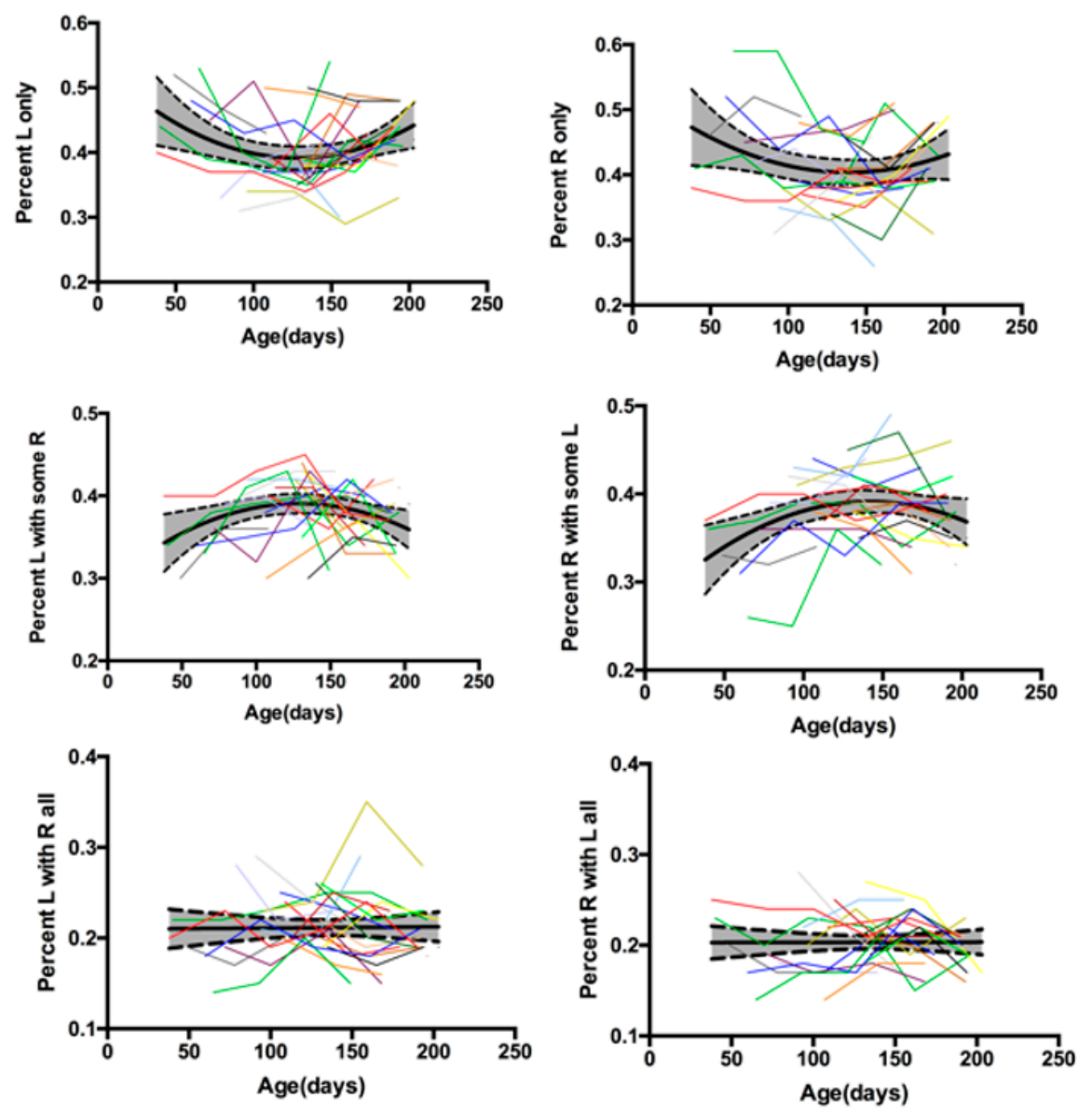

Figure 6. Average percentage of each type of bout (only one arm moving, both arms moving for some portion of the bout, or both arms moving for all of the bout) produced across the full day for the left and right arms, by age, for infants with typical development. Each colored line represents a different infant across 3 to 6 visits. Two single assessments are represented by dots. Thick black line is the mean and shaded area bordered by dashed black line is one standard deviation. Quadratic trends were the best fit for the right and left arms for only one arm moving and both arms moving for some portion of the bout. Linear trends were the best fit for both arms moving for all of the bout.

\subsection{Kinematic Characteristics}

Figure 7 shows the average bout duration (s) at each visit, by age, for infants with TD. A linear trend fit the left and right arm data best, indicating that movement bouts get shorter as infants get older. Figure 8 shows the average acceleration $\left(\mathrm{m} / \mathrm{s}^{2}\right)$ and average peak acceleration $\left(\mathrm{m} / \mathrm{s}^{2}\right)$ at each visit, by age, for infants with TD. For average acceleration, a linear trend fit the left arm data best and a quadratic trend fit the right arm best. For peak acceleration, data for both arms were best fit by a linear 
trend. Taken together, duration and acceleration data indicate that infants move with shorter duration and faster acceleration arm movements as they get older, and the rate of change may not be constant between arms. Table 1 provides the acceleration area values for each arm, at each visit. To compare between infants and across visits, Figure 9 shows the normalized acceleration area for the right and left arms per hour of awake time, by age, for infants with TD. A linear trend fits the right and left arm data best, indicating normalized acceleration area increases as infants get older.
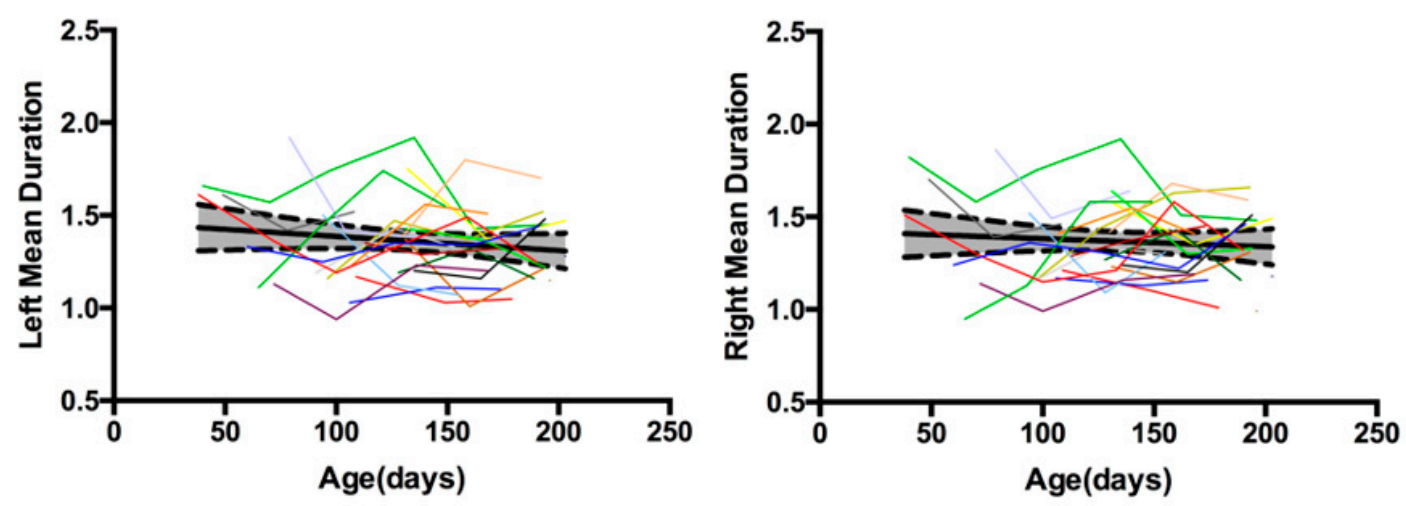

Figure 7. Average bout duration(s) for the left and right arms, by age, for infants with typical development at each visit. Each colored line represents a different infant across 3 to 6 visits. Two single assessments are represented by dots. Thick black line is the mean and shaded area bordered by dashed black line is one standard deviation. A linear trend fit the left and right arm data best.
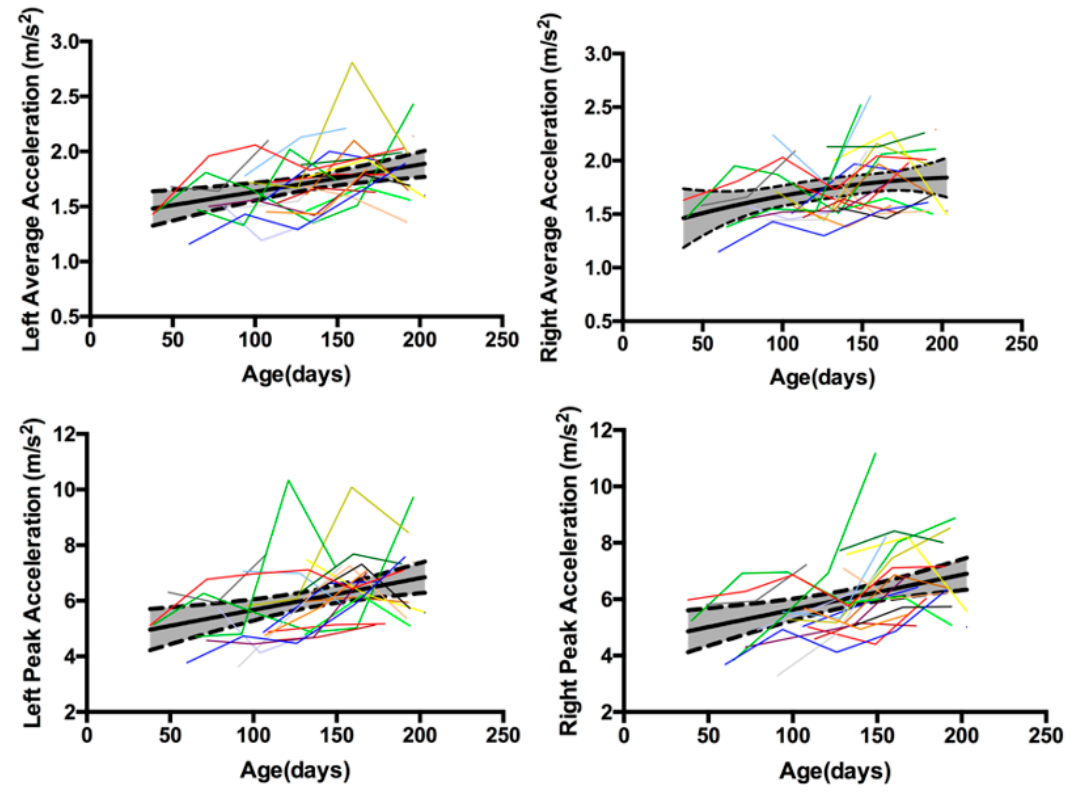

Figure 8. Average acceleration $\left(\mathrm{m} / \mathrm{s}^{2}\right)$ and average peak acceleration $\left(\mathrm{m} / \mathrm{s}^{2}\right)$ for the left and right arms, by age, for infants with typical development at each visit. Each colored line represents a different infant across 3 to 6 visits. Two single assessments are represented by dots. Thick black line is the mean and shaded area bordered by dashed black line is one standard deviation. For left average acceleration, a linear trend fit the data best. For right average acceleration, a quadratic trend fit the data best. Peak acceleration data were best fit by a linear trend. 

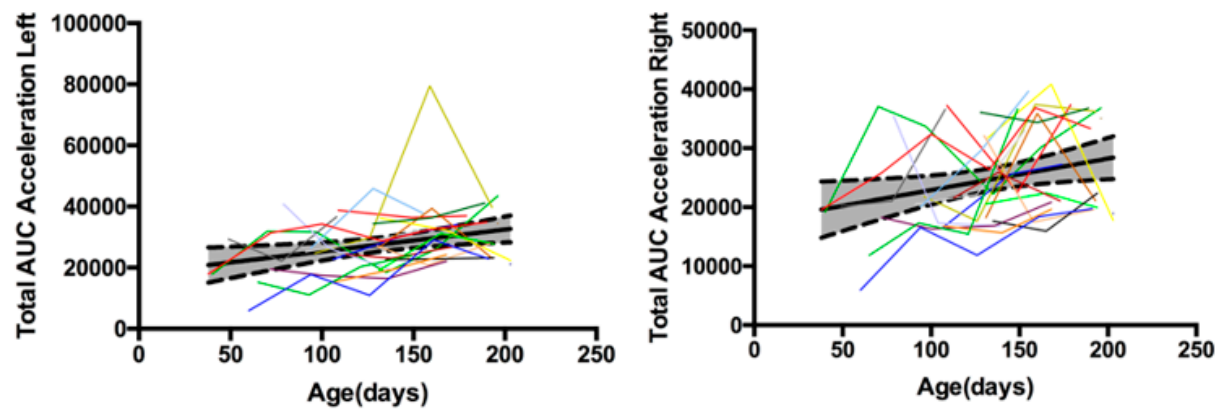

Figure 9. Normalized acceleration area (AUC = area under the curve) for the left and right arms (per hour of awake time), by age, for infants with typical development at each visit. Each colored line represents a different infant across 3 to 6 visits. Two single assessments are represented by dot. Thick black line is the mean and shaded area bordered by dashed black line is one standard deviation. Linear trends fit the right and left arm data best.

\section{Discussion}

Full day infant arm movement monitoring is necessary in order to advance our understanding of how much and what type of movement practice is necessary to learn functional skills. Laboratory based studies are limited to short periods of data collection and do not inform us about how much or how infants are moving across the course of the day in their natural environments. Despite the great knowledge gathered from these studies, it has recently been argued that to further advance the field we now must sample development for a minimum of $24 \mathrm{~h}$ in order to overcome the effects of circadian rhythms, behavioral context, environmental stimuli, mood and motivation, and other factors [19]. In this paper, we move from being able to assess only a few minutes of arm movement data to being able to assess a full day through the use of wearable sensors.

Our purpose was to describe the development of a wearable sensor data algorithm for identifying bouts of infant arm movement in infants with TD and AR. We found an overall performance of the algorithm of $90 \%$ sensitivity. The algorithm does not make systematic errors (consistent over- or under-counting). It is not able to distinguish between the infant moving his or her arms and the caregiver moving the infant's arms (for example, when putting on a shirt), however this type of occurrence is expected to be low among the thousands of bouts of arm movement an infant produces across a day. We feel this amount of non-systematic error is acceptable to move forward with studying how full day movement patterns relate to infant development and the acquisition of functional skills.

Our report here of the number and kinematic characteristics of arm movement bouts from 22 infants with TD is the first step toward developing norms for full-day arm movement behavior. We have provided the values obtained from 8 to $13 \mathrm{~h}$ of arm movement data, as well as normalized to hours of awake time or as a percentage of movements made, in order to allow the comparison between infants and across time. These data are the first step in measuring mean values and variability in a small sample of infants with TD, and will allow us to power future studies to look for relationships between changes in full-day arm movements and functional skill acquisition and to look for early differences in arm movement patterns in infants AR. We have presented data here by chronological age as a first step, however it will be as important to explore movement variables in relation to the developmental trajectory. Two infants of the same chronological age are not expected to be at the same point in their developmental trajectory of motor, cognitive, and/or social development. We believe this method has potential, due to its quantitative assessment and multiple continuous hours of measurement, to embrace the high variability that is a hallmark of typical development and use this information to accurately identify infants who fall outside of the norms of TD very early in development.

Our results for the duration of arm movement bouts averaged around $1.3 \mathrm{~s}$ per bout. Previous literature has focused on the duration of reaches, which are discrete movements of the arm to a target. Infant reach durations have been observed to be approximately $0.6-0.9 \mathrm{~s}$ in 3-month-old infants [20], 
around $0.8 \mathrm{~s}$ in infants from 100 days through 200 days of age and beyond [21], $1.6 \mathrm{~s}$ in 4-month-old infants and $1.2 \mathrm{~s}$ in 6-month-old infants [22], 0.5-0.7 s for 5-month-old infants [23], $1.1 \mathrm{~s}$ for the left arm and $1.3 \mathrm{~s}$ for the right arm in 6-month-old infants [24], and around $1 \mathrm{~s}$ for 7 month-old infants [25]. Given that our arm movement data included both reaching and non-reaching activity, we feel that our results are consistent with the previous data from shorter periods of laboratory-based infant reaching assessments.

There are two studies that include arm acceleration values, each from a single infant. Average arm accelerations across a full day from one infant between 51 to beyond 200 days of age ranged from around 1.2-1.4 m/s $\mathrm{s}^{2}$ [26]. These data included periods of no movement occurring across the day, whereas our data are only during arm movement bouts. As a result of this methodological difference, our average acceleration values are higher (around $1.7 \mathrm{~m} / \mathrm{s}^{2}$ ), but we believe this is reasonable given the different methodology. The other study reported arm peak acceleration of around $6000 \mathrm{~mm} / \mathrm{s}^{2}$ in a 6-month-old boy [24], consistent with our findings of mean peak accelerations around $6 \mathrm{~m} / \mathrm{s}^{2}$.

Across these first months, the majority of the bouts infants made were with one arm only, compared to both arms moving for some or all of the bout. Within that stable preference, however, there appears to be an interesting shift in the relative preference for one arm only moving vs. both arms moving for some portion of the bout. The trajectories indicate that infants start lower but then shift to increase their preference for arm bouts when both arms are moving for some portion of the bout, followed by a decrease. Future work will allow us to explore whether this is related to the development of reaching skill.

Our data are consistent with the previous data, showing that there is not an overwhelming preference across infancy for right or left arm movement; infants with TD employ many different strategies for reaching or otherwise moving their arms. Corbetta and Thelen collected kinematic arm movement data for 4 infants longitudinally from 3 to 52 weeks of age (weekly from 3 to 30 weeks and bi-weekly from 30 to 52 weeks). They identified both reaching and non-reaching interlimb activity. For reaching, preference was attributed to the hand that made initial contact with the target. For laterality in non-reaching movements, the hand with the faster average velocity over a 1-s window was identified as the preferred hand. A right or left preference for each category of movement was determined overall for each visit. The infants produced unstable and fluctuating lateral preferences for reaching and non-reaching movements across the 1st year. Furthermore, when a preference was detected in reaching, it was also observed in non-reaching movements [27]. In another study, 17 infants at 6 months of age did not show a significant difference between right, left, or bimanual reaches, they performed all three types of reaching equally [24].

\section{Conclusions}

We created an algorithm that can be used to quantify kinematic characteristics of infant arm movement bouts produced across a full day in the natural environment. We chose these specific metrics as a first step as they are commonly assessed kinematic measures. In future work, we will explore more advanced computational techniques, such as non-linear analysis measures and machine learning approaches to describe other aspects of our movement data. Furthermore, we will relate the amount and type of arm movement practice across days and months to the development of functional arm reaching skills. Finally, we will determine if early differences in arm movement patterns are predictive of later neuromotor outcomes in infants AR. These results will inform the development of early intervention therapies to support optimal neuromotor development.

Acknowledgments: Thank you to the infants and their families. Thank you to Eisner Pediatric and Medical Center (Los Angeles, CA) and Children's Hospital Los Angeles. This work was supported by the Bill \& Melinda Gates Foundation [OPP1119189] (PI: Smith). Additionally, Smith's salary was supported by NIH K12-HD055929 (PI: Ottenbacher). Lane's effort was supported in part by funding from the National Institutes of Health from the National Center for Advancing Translational Science [UL1TR001855 and UL1TR000130]. The content is solely the responsibility of the authors and does not necessarily represent the official views of the National Institutes of Health. Study data were collected and managed using REDCap electronic data capture tools hosted at the 
Southern California Clinical and Translational Science Institute at the University of Southern California. REDCap (Research Electronic Data Capture) is a secure, web-based application designed to support data capture for research studies, providing (1) an intuitive interface for validated data entry; (2) audit trails for tracking data manipulation and export procedures; (3) automated export procedures for seamless data downloads to common statistical packages; and (4) procedures for importing data from external sources. Trujillo-Priego is supported in part by CONACyT. The Bill \& Melinda Gates Foundation provided funds to cover the cost to publish in open access.

Author Contributions: Beth A. Smith, Douglas L. Vanderbilt, and Gerald E. Loeb conceived and designed the experiments; Beth A. Smith, Ivan A. Trujillo-Priego, and Joanne Shida performed the experiments; Beth A. Smith, Christianne J. Lane, Weiyang Deng, and Ivan A. Trujillo-Priego analyzed the data; Beth A. Smith, Christianne J. Lane, Weiyang Deng, Douglas L. Vanderbilt, Ivan A. Trujillo-Priego, Joanne Shida, and Gerald E. Loeb wrote and edited this manuscript. All authors have read and approved the final manuscript.

Conflicts of Interest: The authors declare no conflict of interest.

\section{References}

1. Hebb, D.O. The Organization of Behavior: A Neuropsychological Theory, 1st ed.; Wiley: New York, NY, USA, 1949.

2. Libertus, K.; Needham, A. Teach to reach: The effects of active vs. passive reaching experiences on action and perception. Vis. Res. 2010, 50, 2750-2757. [CrossRef] [PubMed]

3. Umansky, R. The hand sock, an artificial handicap to prehension in infancy, and its relation to clinical disuse phenomena. Pediatrics 1973, 52, 546-555. [PubMed]

4. Kermoian, R.; Campos, J.J. Locomotor experience: A facilitator of spatial cognitive development. Child Dev. 1988, 59, 908-917. [CrossRef] [PubMed]

5. Schwarzer, G.; Freitag, C.; Buckel, R.; Lofruthe, A. Crawling is associated with mental rotation ability by 9-month-old infants. Infancy 2012, 18, 432-441. [CrossRef]

6. Oudgenoeg-Paz, O.; Volman, M.C.J.M.; Leseman, P.P.M. Attainment of sitting and walking predicts development of productive vocabulary between ages 16 and 28 months. Infant Behav. Dev. 2012, 35, 733-736. [CrossRef] [PubMed]

7. Singh, M.; Patterson, D.J. Involuntary gesture recognition for predicting cerebral palsy in high-risk infants. In Proceedings of the 2010 International Symposium on Wearable Computers (ISWC), Seoul, Korea, 10-13 October 2010; pp. 1-8.

8. Gravem, D.; Singh, M.; Chen, C.; Rich, J.; Vaughan, J.; Goldberg, K.; Waffarn, F.; Chou, P.; Cooper, D.; Reinkensmeyer, D.; et al. Assessment of Infant Movement with a Compact Wireless Accelerometer System. J. Med. Devices 2012, 6, 021013. [CrossRef]

9. Marcroft, C.; Khan, A.; Embleton, N.D.; Trenell, M.; Plötz, T. Movement recognition technology as a method of assessing spontaneous general movements in high risk infants. Front. Neurol. 2015, 5, 284. [CrossRef] [PubMed]

10. Heinze, F.; Hesels, K.; Breitbach-Faller, N.; Schmitz-Rode, T.; Disselhorst-Klug, C. Movement analysis by accelerometry of newborns and infants for the early detection of movement disorders due to infantile cerebral palsy. Med. Biol. Eng. Comput. 2010, 48, 765-772. [CrossRef] [PubMed]

11. Waldmeier, S.; Grunt, S.; Delgado-Eckert, E.; Latzin, P.; Steinlin, M.; Fuhrer, K.; Frey, U. Correlation properties of spontaneous motor activity in healthy infants: A new computer-assisted method to evaluate neurological maturation. Exp. Brain Res. 2013, 227, 433-446. [CrossRef] [PubMed]

12. Ohgi, S.; Morita, S.; Loo, K.K.; Mizuike, C. Time Series Analysis of Spontaneous Upper-Extremity Movements of Premature Infants with Brain Injuries. Phys. Ther. 2008, 88, 1022-1033. [CrossRef] [PubMed]

13. Gima, H.; Ohgi, S.; Morita, S.; Karasuno, H.; Fujiwara, T.; Abe, K. A Dynamical System Analysis of the Development of Spontaneous Lower Extremity Movements in Newborn and Young Infants. J. Physiol. Anthropol. 2011, 30, 179-186. [CrossRef] [PubMed]

14. Activity Monitor Comparison I ActiGraph. Available online: actigraphcorp.com/products-showcase/ activity-monitors/product-comparison/ (accessed on 24 July 2016).

15. Actical Specifications. Available online: http://www.actigraphy.com/solutions/actical/specifications.html (accessed on 22 July 2017).

16. Bayley, N. Bayley Scales of Infant Development, 3rd ed.; Pearson: San Antonio, TX, USA, 2005.

17. California Department of Health Care Services. Available online: http://www.dhcs.ca.gov/services/ccs / Pages/HRIF.aspx\#medicalcriteria (accessed on 20 May 2017). 
18. Piper, M.C.; Darrah, J. Motor Assessment of the Developing Infant; WB Saunders: Philadelphia, PA, USA, 1994.

19. Adolph, K.E.; Robinson, S.R. Sampling Development. J. Cogn. Dev. 2011, 12, 411-423. [CrossRef] [PubMed]

20. Cunha, A.B.; de Almeida Soares, D.; de Paula Carvalho, R.; Rosander, K.; von Hofsten, C.; Tudella, E. Maturational and situational determinants of reaching at its onset. Infant Behav. Dev. 2015, 41, 64-72. [CrossRef] [PubMed]

21. Berthier, N.E.; Keen, R. Development of reaching in infancy. Exp. Brain Res. 2005, 169, 507-518. [CrossRef] [PubMed]

22. Fallang, B.; Saugstad, O.D.; Grøgaard, J.; Hadders-Algra, M. Kinematic Quality of Reaching Movements in Preterm Infants. Pediatr. Res. 2003, 53, 836-842. [CrossRef] [PubMed]

23. Fetters, L.; Todd, J. Quantitative Assessment of Infant Reaching Movements. J. Mot. Behav. 1987, 19, 147-166. [CrossRef] [PubMed]

24. Rönnqvist, L.; Domellöf, E. Quantitative assessment of right and left reaching movements in infants: A longitudinal study from 6 to 36 months. Dev. Psychobiol. 2006, 48, 444-459. [CrossRef] [PubMed]

25. Tronick, E.Z.; Fetters, L.; Olson, K.L.; Chen, Y. Similar and functionally typical kinematic reaching parameters in 7- and 15-month-old in utero cocaine-exposed and unexposed infants. Dev. Psychobiol. 2004, 44, 168-175. [CrossRef] [PubMed]

26. Abney, D.H.; Warlaumont, A.S.; Haussman, A.; Ross, J.M.; Wallot, S. Using nonlinear methods to quantify changes in infant limb movements and vocalizations. Front. Psychol. 2014, 5, 771. [CrossRef] [PubMed]

27. Corbetta, D.; Thelen, E. Lateral biases and fluctuations in infants' spontaneous arm movements and reaching. Dev. Psychobiol. 1999, 34, 237-255. [CrossRef]

(C) 2017 by the authors. Licensee MDPI, Basel, Switzerland. This article is an open access article distributed under the terms and conditions of the Creative Commons Attribution (CC BY) license (http:// creativecommons.org/licenses/by/4.0/). 